\title{
F8-IL10: A New Potential
}

\section{Antirheumatic Drug Evaluated by a PET-Guided Translational Approach}

\section{Journal Article}

\section{Author(s):}

Bruijnen, Stefan T.G.; Chandrupatla, Durga M.S.H; Giovanonni, Leonardo; Neri, Dario (D); Vugts, Danielle J.; Huisman, Marc C.; Hoekstra, Otto S.; Musters, René J.P.; Lammertsma, Adriaan A.; van Dongen, Guus A. M. S.; Jansen, Gerrit; Molthoff, Carla F.M.; van der Laken, Conny J.

Publication date:

2019-01-07

\section{Permanent link:}

https://doi.org/10.3929/ethz-b-000315346

\section{Rights / license:}

Creative Commons Attribution-NonCommercial-NoDerivatives 4.0 International

Originally published in:

Molecular Pharmaceutics 16(1), https://doi.org/10.1021/acs.molpharmaceut.8b00982 


\title{
F8-IL10: A New Potential Antirheumatic Drug Evaluated by a PET- Guided Translational Approach
}

\author{
Stefan T.G. Bruijnen, ${ }^{\dagger}$ (® Durga M.S.H. Chandrupatla, ${ }^{\dagger}$ Leonardo Giovanonni, ${ }^{\S, \# ~ D a r i o ~ N e r i, ~}{ }^{\|}$
}

Danielle J. Vugts, ${ }^{\ddagger}$ Marc C. Huisman, ${ }^{\ddagger}$ Otto S. Hoekstra, ${ }^{\ddagger}$ René J.P. Musters, ${ }^{\perp}$ Adriaan A. Lammertsma, ${ }^{\ddagger}$ Guus A.M.S. van Dongen, ${ }^{\dagger}$ Gerrit Jansen, ${ }^{\dagger}$ Carla F.M. Molthoff, ${ }^{\dagger}$ and Conny J. van der Laken ${ }^{*}{ }^{\dagger}$

${ }^{\dagger}$ Amsterdam Rheumatology and Immunology Center, Amsterdam University Medical Center, Location VU University Medical Center, 1007 MB Amsterdam, The Netherlands

${ }^{*}$ Department of Radiology \& Nuclear Medicine, ${ }^{\perp}$ Department of Physiology, Amsterdam University Medical Center, location VU University Medical Center, 1007 MB Amsterdam, The Netherlands

${ }^{\S}$ Philogen S.p.A., 53100 Siena, Italy

"Institute of Pharmaceutical Sciences, ETH Zürich, 8092 Zürich, Switzerland

\section{Supporting Information}

ABSTRACT: Antibody fragment F8-mediated interleukin 10 (IL10) delivery is a novel treatment for rheumatoid arthritis (RA). F8 binds to the extra-domain-A of fibronectin (ED-A). In this study, in vivo biodistribution and arthritis targeting of radiolabeled F8-IL10 were investigated in RA patients, followed by further animal studies. Therefore, three RA patients (DAS28 > 3.2) received $0.4 \mathrm{mg}$ of $30-74$ megabecquerel $\left[{ }^{124} \mathrm{I}\right] \mathrm{I}-\mathrm{F} 8-\mathrm{IL} 10$ for PET-CT and blood sampling. In visually identified PET-positive joints, target-tobackground was calculated. Healthy mice, rats, and arthritic rats were injected with iodinated F8-IL10 or KSF-IL10 control antibody. Various organs were excised, weighed, and counted for radioactivity. Tissue sections were stained for fibronectin ED-A. In RA patients, $\left[{ }^{124} \mathrm{I}\right] \mathrm{I}-\mathrm{F} 8-\mathrm{IL} 10$ was cleared rapidly from the circulation with less than $1 \%$ present in blood after 5 min. PET-CT showed targeting in 38 joints (11-15 per patient) and high uptake in the liver and spleen. Mean target-tobackground ratios of PET-positive joints were $2.5 \pm 1.2$, 1.5 times higher for clinically active than clinically silent joints. Biodistribution of radioiodinated F8-IL10 in healthy mice showed no effect of the radioiodination method. [ $\left.{ }^{124} \mathrm{I}\right] \mathrm{I}-\mathrm{F} 8-\mathrm{IL} 10$ joint uptake was also demonstrated in arthritic rats, $\sim 14$-fold higher than that of the control antibody [ $\left.{ }^{124} \mathrm{I}\right] \mathrm{I}-\mathrm{KSF}-\mathrm{IL} 10$ ( $p<$ $0.001)$. Interestingly, liver and spleen uptake were twice as high in arthritic than in healthy rats and were related to increased $(\sim 7 \times)$ fibronectin ED-A expression in these tissues. In conclusion, $\left[{ }^{124} \mathrm{I}\right] \mathrm{I}-\mathrm{F} 8-\mathrm{IL} 10$ uptake was observed in arthritic joints in RA patients holding promise for visualization of inflamed joints by PET-CT imaging and therapeutic targeting. Patient observations and, subsequently, arthritic animal studies pointed to awareness of increased [ $\left.{ }^{124} \mathrm{I}\right] \mathrm{I}-\mathrm{F} 8-\mathrm{IL} 10$ uptake in the liver and spleen associated with moderate systemic inflammation. This translational study demonstrated the value of in vivo biodistribution and PET-CT-guided imaging in development of new and potential antirheumatic drugs'.

KEYWORDS: fibronectin (ED-A), IL10, rheumatoid arthritis, pharmacokinetics, positron emission tomography, computed tomography

\section{INTRODUCTION}

Rheumatoid arthritis (RA) is a chronic inflammatory and destructive joint disease ${ }^{1}$ for which treatment options currently include chemical and biological disease modifying antirheumatic drugs (DMARDs). ${ }^{2,3}$ Nevertheless, $30-40 \%$ of patients are refractory to these treatments, leaving an unmet need for alternative therapies. ${ }^{4}$ Targeted delivery of cytokines at the site of disease is an emerging novel approach to treat chronic inflammation, especially $\mathrm{RA}^{5}$ and has also been successfully investigated in the cancer setting over the past few years. ${ }^{6-9}$ Immunocytokines (cytokine-antibody fusion proteins) repre- sent a class of therapeutic agents that have previously shown their usefulness for targeting antigens at the site of inflammation followed by local activity of the cytokine. ${ }^{5}$ Along these lines, target specificity and dose-mediated therapy response can be achieved, especially at synovium in RA.

Received: September 18, 2018

Revised: November 27, 2018

Accepted: December 14, 2018

Published: December 14, 2018 
Interleukin 10 (IL10) is an anti-inflammatory cytokine, produced by activated monocytes and $\mathrm{T}$ cells, and is involved in the regulation of inflammatory responses and immune reactions. ${ }^{10,11}$ In the clinic, combination therapy of IL10 and methotrexate in a multicenter, placebo-controlled study in RA patients revealed clinical American College of Rheumatology 20 responses in $50-60 \%$ of patients compared with $10 \%$ for placebo. ${ }^{12}$ Despite the anti-inflammatory properties of IL10, systemic administration in RA patients generated insufficient responses to pursue further development. However, when IL10 is fused with the single-chain antibody variable domain $(\mathrm{Fv})$ fragment of antibody F8, targeted delivery of IL10 can be achieved locally, especially at RA synovium. Antibody fragment F8 binds to the extra-domain A (ED-A) of fibronectin, selectively expressed at sites of inflammation in $\mathrm{RA}^{13}$ and in tumors ${ }^{14}$ in humans as well as in animals. Preclinical studies show that F8 binds with comparable affinity to ED-A of murine, monkey, and human origin. ${ }^{15}$ Previously, high levels of fibronectin ED-A have been demonstrated in the target tissues of arthritic as well as tumor-bearing animal models. ${ }^{15,96}$ Besides, F8-IL10 displayed clear localization at sites of arthritis in a collagen-induced arthritis mouse model. ${ }^{16}$ Recently, encouraging early signals of the therapeutic benefit of F8-IL10 were demonstrated in RA patients in a phase I study, underscoring the therapeutic potential of this new approach. ${ }^{17}$ Positron emission tomography (PET) can provide unique whole-body molecular information about targeting and in vivo biodistribution of new therapeutic agents. ${ }^{18,19}$ For imaging of antibodies, isotopes with long half-lives as iodine-124 $\left({ }^{124} \mathrm{I}\right)$ and zirconium-89 $\left({ }^{89} \mathrm{Zr}\right)$ allow for imaging of targeting and in vivo biodistribution over several days. ${ }^{20-22}$ The purpose of the present translational study was to investigate the in vivo distribution and arthritis targeting of $\left[{ }^{124} \mathrm{I}\right] \mathrm{I}-\mathrm{F} 8-\mathrm{IL} 10$ in RA patients, followed by animal studies.

\section{METHODS}

Patients. Three RA patients (aged $\geq 18$ years) who fulfilled the American College of Rheumatology 1987 classification criteria for rheumatoid arthritis ${ }^{23}$ were included during 2013 and 2014 (Table 1). Patients eligible for this study required a high disease activity score across 28 joints (DAS28) of $\geq 3.2$ and $\geq 2$ clinically inflamed joints in hands. Exclusion criteria consisted of any hematologic, liver, or renal function test abnormalities; concurrent active infections/autoimmune disease or severe (malignant) diseases (other than RA); immunodeficiency or human immunodeficiency virus positivity; (history of) heart problems or insufficiency; uncontrolled hypertension; pregnancy or breastfeeding; major surgery within the previous 4 weeks; or any other treatment with investigational drugs within the previous three months. Stable doses of DMARDs and/or nonsteroidal anti-inflammatory drugs (NSAIDs) were continued if used at inclusion. All clinical data were obtained by an experienced research nurse blinded to the imaging data.

Animals. Wistar rats (male, 150-200 g, Charles River International Inc., Sulzfeld, Germany) and healthy mice (6-8 weeks female, Envigo, Horst, NL) were provided with standard food (16\% protein rodent diet, Harlan Laboratories Inc., Madison, WI, USA) and water ad libitum. Rats were housed in conventional cages and mice in filter top cages (in groups of 3-6 per cage) and kept in a room with a 12-h light/dark cycle and constant room temperature $\left(21^{\circ} \mathrm{C}\right)$ and humidity level (55 $\pm 10 \%)$.
For arthritis induction, Wistar rats were immunized systemically and additionally received $4 \times$ intra-articular methylated bovine serum albumin injections, 4 or 5 days apart, in the arthritic (right) knee, with the contralateral (left) knee serving as a control, as described previously. ${ }^{24,25}$

Tracer Synthesis. The full description of the tracer synthesis for administration to RA patients, mice, and rats is presented in Appendix A in the Supporting Information. Essentially, F8-IL10 was obtained from Philogen (Zurich, Switzerland) and was iodinated with iodine- 124 for humans, rats, and mice, and iodine-131 for mice with a radiochemical purity of $>95 \%$, according to instant thin layer chromatography (iTLC) and high-performance liquid chromatography (HPLC). The immune reactive fraction was determined by measuring binding to a serial dilution of ED-A-coated beads, essentially, as described by Lindmo et al. ${ }^{26}$ For the study in healthy mice, apart from labeling of F8-IL10 with ${ }^{131} \mathrm{I}$ according to the iodogen method (as was done with labeling for humans and rats), F8-IL10 was also labeled with ${ }^{124} \mathrm{I}$ according to the chloramine-T method used earlier by Philogen. ${ }^{27}$ Radiolabeling of F8-IL10 and control antibody KSF-IL10 with ${ }^{124}$ I was applied in all experiments with rats, essentially, according to Tijink et al. ${ }^{28} \mathrm{KSF}$ is an antibody recognizing hen egg lysozyme and has no binding specificity in rats. $^{29}$

[ ${ }^{124}$ I]I-F8-IL10 PET-CT of RA Patients. Before PET-CT scanning, patients received prophylaxis to block the thyroid by administration of $400 \mathrm{mg}$ of potassium perchlorate 48,24 , and 1 $\mathrm{h}(\mathrm{s})$ before infusion of $\left.{ }^{124} \mathrm{I}\right] \mathrm{I}-\mathrm{F} 8-\mathrm{IL} 10$ and $1 \mathrm{~h}$ before PETCT scanning at 24 and $72 \mathrm{~h}$. Next, a total of 3 whole body PETCT scans per patient and detailed images of wrists/hands were obtained, and blood samples were withdrawn at 5, 30, $90 \mathrm{~min}, 3$, 24 , and $72 \mathrm{~h}$ post injection (p.i.).

PET-CT scans were performed using both Gemini-64 and Ingenuity-128 PET-CT scanners (Philips Healthcare, Cleveland, USA). After intravenous (i.v.) injection of a single dose of $\left[{ }^{124} \mathrm{I}\right] \mathrm{I}-\mathrm{F} 8-\mathrm{IL} 10(0.4 \mathrm{mg}(\sim 6 \mu \mathrm{g} / \mathrm{kg}), 59 \pm 25 \mathrm{MBq})$, patients were scanned at $1,20-22.5$, and $69-76 \mathrm{~h}$ p.i.. The administration system was flushed with $20 \mathrm{~mL}$ of $0.9 \% \mathrm{NaCl}$, and after administration of the tracer, residual activity in the injection device was measured to determine the net injected dose. Subsequently, whole body scans from skull to symphysis pubis were acquired with $5 \mathrm{~min} /$ field of view. In addition, detailed images of wrists/hands were obtained ( 1 field of view, 6 frames of $5 \mathrm{~min}$ each). PET scans were preceded by a $30 \mathrm{mAs}$ low-dose CT for attenuation correction and anatomical localization of the PET signal.

PET data were normalized and corrected for attenuation, decay, and scatter. All scans were reconstructed according to international guidelines. ${ }^{30}$

Image Analysis. An experienced nuclear medicine physician (O.S. Hoekstra) qualitatively interpreted PET-CT data for whole body biodistribution and joints with visually enhanced tracer uptake (PET-positive joints). For quantitative comparison of tracer uptake, volumes of interest (VOIs) were drawn using software developed in-house, ${ }^{31}$ with the corresponding low dose CT as an anatomical reference. For biodistribution, VOIs were drawn over lungs, liver, spleen, kidneys, heart (blood pool), and vertebrae. This was performed using in-house standard operating procedures by drawing VOIs over the entire organ (CT as reference), and results were presented as percentage of the injected dose (\%ID).

For analysis of visually defined PET-positive joints, VOIs were drawn on top of joints with clear focal tracer enhancement using 
thresholds exceeding local background uptake (e.g., PET negative joints). In addition, for PET-negative hand joints, standardized spherical VOIs were drawn on wrists $(\sim 60 \mathrm{~mL})$, metacarpophalangeal joints $1-5(8 \mathrm{~mL})$, and (proximal) interphalangeal joints $1-5(2.0 \mathrm{~mL})$ centered in the middle of the joints ( $n=22$ joints per patient). Standardized uptake values were calculated by dividing the PET tracer tissue concentration by the activity injected (MBq) per body weight (kg). Tracer uptake in targets is presented as $\mathrm{SUV}_{\text {peak }}$ which is defined as the highest average uptake within a sphere of $1.2 \mathrm{~mL}$ within the VOI. ${ }^{32,33}$ In addition, spherical VOIs of $0.5 \mathrm{~mL}$ in the second metacarpal bone were used as background to calculate target-tobackground ratios.

Animal Studies. Ex Vivo Biodistribution of $\left[{ }^{124} I /{ }^{131} I\right] I-F 8-$ IL 10 in Healthy Mice Comparing Radio-lodination Methods. Healthy mice $(n=14)$ were injected with either $\left[{ }^{124} \mathrm{I}\right] \mathrm{I}-\mathrm{F} 8-$ IL10 (chloramine-T method) or $\left[{ }^{131} \mathrm{I}\right] \mathrm{I}-\mathrm{F} 8-\mathrm{IL} 10$ (iodogen method) at $10 \mathrm{~min}(n=3)$ and $24 \mathrm{~h}(n=4)$. In both cases, the F8-IL10 protein dose was $3 \mathrm{mg} / \mathrm{kg}$. Each mouse received $\sim 3.5$ MBq $\left[{ }^{124} \mathrm{I}\right] \mathrm{I}-\mathrm{F} 8-\mathrm{IL} 10$ or $\left[{ }^{131} \mathrm{I}\right] \mathrm{I}-\mathrm{F} 8-\mathrm{IL} 10$.

At the end of the study, all mice were sacrificed, and blood and various tissues were excised, rinsed, dipped dry, and weighed. The amount of tissue radioactivity was determined using an LKB 1282 Compugamma CS gamma counter (LKB, Wallac, Turku, Finland). Results were expressed as a percentage of the injected dose per gram tissue $(\% \mathrm{ID} / \mathrm{g})$. $^{24}$

Ex Vivo Biodistribution of $\left[{ }^{124} I\right] I-F 8-I L 10$ in Healthy and Arthritic Rats. Iodine-124 was used in the rat experiments to allow for a better and direct comparison of findings in rat experiments and human studies. Six days after the last intraarticular injection of methylated bovine serum albumin, all rats received $\sim 3.5 \mathrm{MBq}$ tracer injection (summarized in Table 1).

Table 1. Tracers and Dosages Used in Healthy/Arthritic Rat Studies

\begin{tabular}{|c|c|c|}
\hline & tracer & $\begin{array}{l}\text { dosage } \\
(\mathrm{mg} / \mathrm{kg})\end{array}$ \\
\hline group A: healthy rats $\left(\mathrm{F} 8-\mathrm{IL} 10^{\text {low }} ; n=4\right)$ & 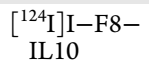 & 0.35 \\
\hline $\begin{array}{l}\text { group B: arthritic rats control (KSF-IL10; } \\
n=6 \text { ) }\end{array}$ & $\begin{array}{l}{\left[\begin{array}{l}\left.{ }^{124} \mathrm{I}\right] \mathrm{I}-\mathrm{KSF}- \\
\mathrm{IL} 10\end{array}\right.}\end{array}$ & 0.35 \\
\hline group C: arthritic rats $\left(\mathrm{F} 8-\mathrm{IL} 10^{\text {low }} ; n=6\right)$ & $\begin{array}{l}{\left[{ }^{124} \mathrm{I}\right] \mathrm{I}-\mathrm{F} 8-} \\
\mathrm{IL} 10\end{array}$ & 0.35 \\
\hline group D: arthritic rats $\left(\mathrm{F} 8-\mathrm{IL} 10^{\text {hgh }} ; n=6\right)$ & $\begin{array}{l}{\left[{ }^{124} \mathrm{I}\right] \mathrm{I}-\mathrm{F} 8-} \\
\quad \mathrm{IL} 10\end{array}$ & 12.9 \\
\hline
\end{tabular}

The control and low dose groups receiver was $3.5 \mathrm{MBq} / 100 \mu \mathrm{g}$, and the high dose group was $3.5 \mathrm{MBq} / 3.75 \mathrm{mg}$. $24 \mathrm{~h}$ post tracer injection; all rats were sacrificed, and knee joints, as well as various tissues, were removed and analyzed as described above in the mice experiment.

Immunofluorescence of Liver and Spleen of Arthritic versus Healthy Rats. Tissues (liver and spleen) of arthritic rats $(n=4)$ and healthy rats $(n=4)$ were snap frozen in liquid nitrogen and stored at $-80{ }^{\circ} \mathrm{C}$. Sections of $8 \mu \mathrm{m}$ were cut and stained with hematoxylin and eosin (HE), whereas staining of splice isoforms of fibronectin ED-A was performed by F8-small immunoprotein (SIP) (Philogen, Siena, Italy) or isotype control antibody. Tissues were embedded in a suitable medium (OCT; SKU4583, Tissue-Tek, Netherlands), cut using a cryotome cryostat $\left(-20{ }^{\circ} \mathrm{C}\right)$ (Leica, Netherlands), and placed on Superfrost (4951PLUS4, ThermoFisher, Netherlands) glass slides for immunofluorescence staining.
For immunostaining, frozen tissue sections were first brought to room temperature for $30 \mathrm{~min}$. The sections were fixed in acetone (439126, Sigma-Aldrich, Netherlands) for $10 \mathrm{~min}$ at $-20^{\circ} \mathrm{C}$ and air-dried for $10 \mathrm{~min}$ at room temperature. Sections were marked with a DAKO pen (S2002, DAKO, Santa Clara, CA, USA). After being washed $3 \times$ with PBS on a shaker, sections were treated with $100 \%$ fetal bovine serum (FBS) for $30 \mathrm{~min}$ at room temperature to avoid nonspecific binding and then washed again with PBS $(3 \times 5 \mathrm{~min})$. Thereafter, sections were incubated with F8-small immunoprotein (SIP) $(2 \mu \mathrm{g} / \mathrm{mL})$ in $10 \% \mathrm{FBS} /$ PBS for $24 \mathrm{~h}$ at $4{ }^{\circ} \mathrm{C}$ or with $10 \% \mathrm{FBS} / \mathrm{PBS}$. After a washing step ( $3 \times 5$ min in PBS on a shaker), sections were incubated with rabbit anti-human IgE 1:1000 in 10\% FBS/PBS (DAKO, A0094, Santa Clara, United States) for $60 \mathrm{~min}$ at room temperature. After a final washing step, sections were incubated with goatanti-rabbit Alexa 488 (A-11008, ThermoFisher Scientific, Netherlands) in 10\% FBS/PBS), air-dried, and mounted (2 $\mu \mathrm{L}$ of MOWIOL mounting medium (81381, Merck, Zwijndrecht, The Netherlands).

Microscopy and Image Analysis of Immunofluorescent Tissue Sections. The slides were imaged with a Zeiss Axiovert 200 M Marianas inverted microscope ( $40 \times$ oil-immersion lens), equipped with a motorized stage (stepper-motor $z$-axis increments: $0.1 \mu \mathrm{m}$ ), and a turret of four epifluorescence cubes (fluorescein isothiocyanate, Cy-5, Cy-3, aminomethylcoumarin, as well as a differential interference contrast bright field cube). A cooled charge-coupled device camera (Cooke Sensicam SVGA [Cooke Co., Tonawanda, NY, USA], $1280 \times 1024$ pixels) recorded images with true 16-bit capability. The camera was linear over its full dynamic range (up to intensities of over 4000) with dark/background currents (estimated by the intensity outside the cells) of typically $<100$. Exposure, objective, montage, and pixel binning were automatically recorded and stored with each image (Dell Dimension workstation: Quadcore processor, 16GB RAM). The microscope, camera, and data processing were controlled by SlideBook software (SlideBook version 6, Intelligent Imaging Innovations, Denver, CO, USA). ${ }^{34}$

All images obtained in 2D were first deconvolved (no neighbor) in order to improve the signal-to-noise ratio. The arthritic and healthy liver/spleen images were segment masked (using background from negative liver/spleen sections). Thereupon, mask statistics were performed on the entire mask, and the sum intensities were recorded. Sum intensities of all multiple representative images from arthritic and healthy rat liver/spleen were averaged $( \pm S D)$.

Histology images were captured using a Leica 4000B microscope and a Leica digital camera DC500 (Microsystems B.V. Rijswijk, The Netherlands).

Statistical Analysis. PET data of RA patients were reported in a descriptive manner because of the small sample size. Quantitative human and RA-rat model data are presented as the mean \pm standard deviation (SD) or as median and interquartile range (IQR), in the case of skewed distribution. Mann-Whitney $\mathrm{U}$ (exact) tests were performed to analyze differences in tissue distribution and fibronectin ED-A staining between different rat groups $(A-D)$. A $p$-value $<0.05$ was considered as statistically significant. Statistical analyses were performed using SPSS version 22.0 for Windows (SPSS, Chicago, IL, USA). 


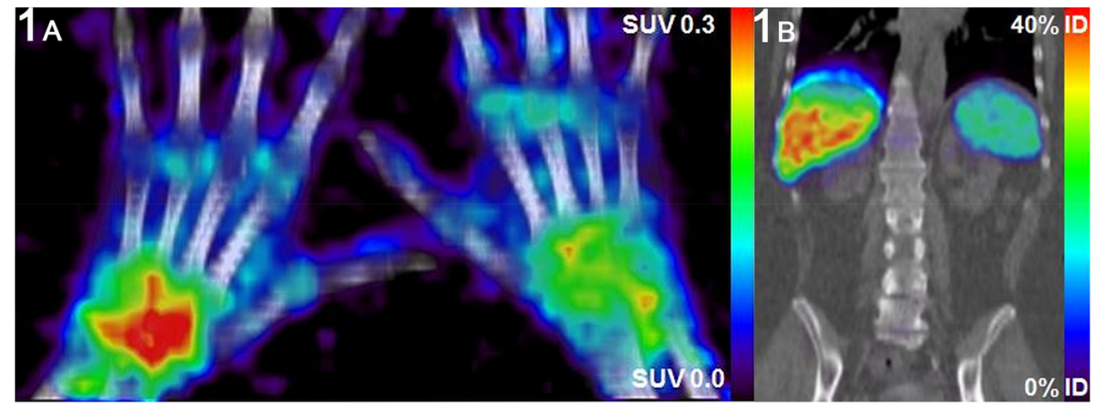

Figure 1. (A) Example of a $\left[{ }^{124} \mathrm{I}\right] \mathrm{I}-\mathrm{F} 8-\mathrm{IL} 10$ PET-CT scan of the hands of RA patient 3 with clinically active disease. (B) [ $\left.{ }^{124} \mathrm{I}\right] \mathrm{I}-\mathrm{F} 8-\mathrm{IL} 10 \mathrm{PET}$ scan of RA patient 1 showing clear tracer uptake in liver and spleen. \%ID = percentage of the injected dose.

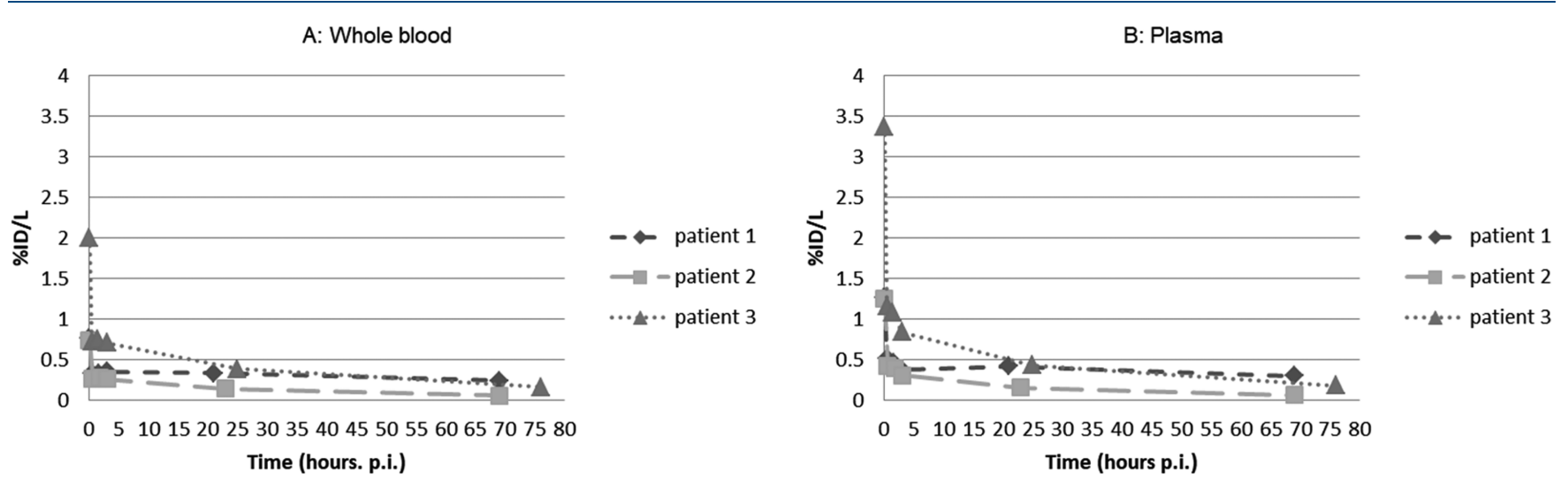

Figure 2. Pharmacokinetic analysis of $\left[{ }^{124} \mathrm{I}\right] \mathrm{I}-\mathrm{F} 8-\mathrm{IL} 10$ in (A) whole blood and (B) plasma of three RA patients.

\section{RESULTS}

Clinical Data of RA Patients. Clinical characteristics of the three patients included are summarized in Appendix B, Supporting Information.

All patients had high disease activity despite stable doses of DMARDs, with a DAS28 $>4.0$. Out of 66 evaluated hand/wrist joints of RA patients, 25 were clinically tender and/or swollen (38\%), i.e., 5, 15, and 5 in patients 1,2 , and 3, respectively. Clinical symptoms of arthritis (tenderness and/or swelling) were present in wrists $(5 / 66 ; 8 \%)$, metacarpophalangeal joints $(15 / 66 ; 22 \%)$, and proximal phalangeal joints $(5 / 66 ; 8 \%)$. $\left[{ }^{124} \mathrm{I}\right] \mathrm{I}-\mathrm{F} 8-\mathrm{IL} 10$ was injected without any side effects occurring during the observation period.

Joint Targeting of $\left[{ }^{124} \mathrm{I}\right] \mathrm{I}-\mathrm{F} 8-\mathrm{IL} 10$ in RA Patients. Detailed images of wrists/hands showed evident targeting in joints (Figure 1A). The highest uptake and target-to-background ratios were determined at $24 \mathrm{~h}$ p.i. Within the one field of view of the hands, a total of 57 hand joints across the 3 patients could be imaged using $\left[{ }^{124} \mathrm{I}\right] \mathrm{I}-\mathrm{F} 8-\mathrm{IL} 10$, and a cumulative number of 38 PET-positive joints were observed (11-5 joints per patient). Out of $38\left[{ }^{124} \mathrm{I}\right] \mathrm{I}-\mathrm{F} 8-\mathrm{IL} 10$ PET-positive joints, 19 were also clinically swollen and/or tender (50\%). In addition, there was enhanced uptake in 19 hand joints that lacked clinical signs of arthritis. The remaining 19/57 joints were PETnegative, of which 17 (89\%) joints were also clinically negative.

The mean standard uptake value of PET-positive joints was $0.25 \pm 0.15$, and the mean target-to-background ratio was $2.5 \pm$ 1.2. PET-positive joints that were also clinically active had 1.5fold higher target-to-background ratios than those of clinically silent PET-positive joints (i.e., subclinical joints) (target-tobackground ratios $2.6 \pm 1.2$ vs $1.6 \pm 0.8$, respectively).
Whole-Body Distribution of $\left[{ }^{124}\right.$ I]I-F8-IL10 in RA Patients. Blood sample analysis in RA patients indicated very fast clearance of $\left[{ }^{124} \mathrm{I}\right] \mathrm{I}-\mathrm{F} 8-\mathrm{IL} 10$ from the circulation after i.v. injection of the drug. In fact, after $5 \mathrm{~min}$, less than $\sim 1 \%$ of the injected dose was detected in blood (Figure 2). Another remarkable finding was the high uptake of $\left[{ }^{124} \mathrm{I}\right] \mathrm{I}-\mathrm{F} 8-\mathrm{IL} 10$ in liver $(48.0 \pm 3.5 \%$ of the injected dose, \%ID) and, to a lower extent, spleen $(5.3 \pm 3.5 \% \mathrm{ID})$, as seen in the whole body PETCT scans performed at $1 \mathrm{~h}$ p.i. (Table 2, Figure 1B). Thereafter, $\left[{ }^{124} \mathrm{I}\right] \mathrm{I}-\mathrm{F} 8-\mathrm{IL} 10$ liver uptake decreased from 48.0 (\%ID) to 13.4 (\%ID) at $72 \mathrm{~h}$ p.i. (Table 2).

Table 2. Organ Uptake of $\left[{ }^{124} \mathrm{I}\right] \mathrm{I}-\mathrm{F} 8-\mathrm{IL} 10$ Expressed as a \% ID 1 and $72 \mathrm{~h}$ p.i.

\begin{tabular}{lcc} 
& \%ID at 1 h p.i. & \%ID at 72 h p.i. \\
& mean \pm SD & mean \pm SD \\
heart (blood pool) & $0.005 \pm 0.002$ & $0.001 \pm 0.0007$ \\
kidneys & $1.5 \pm 0.4$ & $0.5 \pm 0.3$ \\
liver & $48.0 \pm 3.5$ & $13.4 \pm 2.7$ \\
spleen & $5.3 \pm 3.5$ & $1.4 \pm 1.5$ \\
bone marrow & $0.2 \pm 0.1$ & $0.004 \pm 0.001$ \\
\hline
\end{tabular}

To further understand the high uptake in liver and spleen, animal experiments were performed.

Effect of Radioiodination on the in Vivo Biodistribution of F8-IL10 in Healthy Mice. To investigate whether radioiodination affected F8-IL10, resulting in increased uptake in liver and spleen, healthy mice were analyzed for biodistribution after i.v. injection of F8-IL10 labeled according to the chloramine-T ([$\left.\left.{ }^{124} \mathrm{I}\right] \mathrm{I}-\mathrm{F} 8-\mathrm{IL} 10\right)$ and iodogen method ([ $\left.\left.{ }^{131} \mathrm{I}\right] \mathrm{I}-\mathrm{F} 8-\mathrm{IL} 10\right)$, respectively (Figure 3 ). At $10 \mathrm{~min}$ p.i., both 


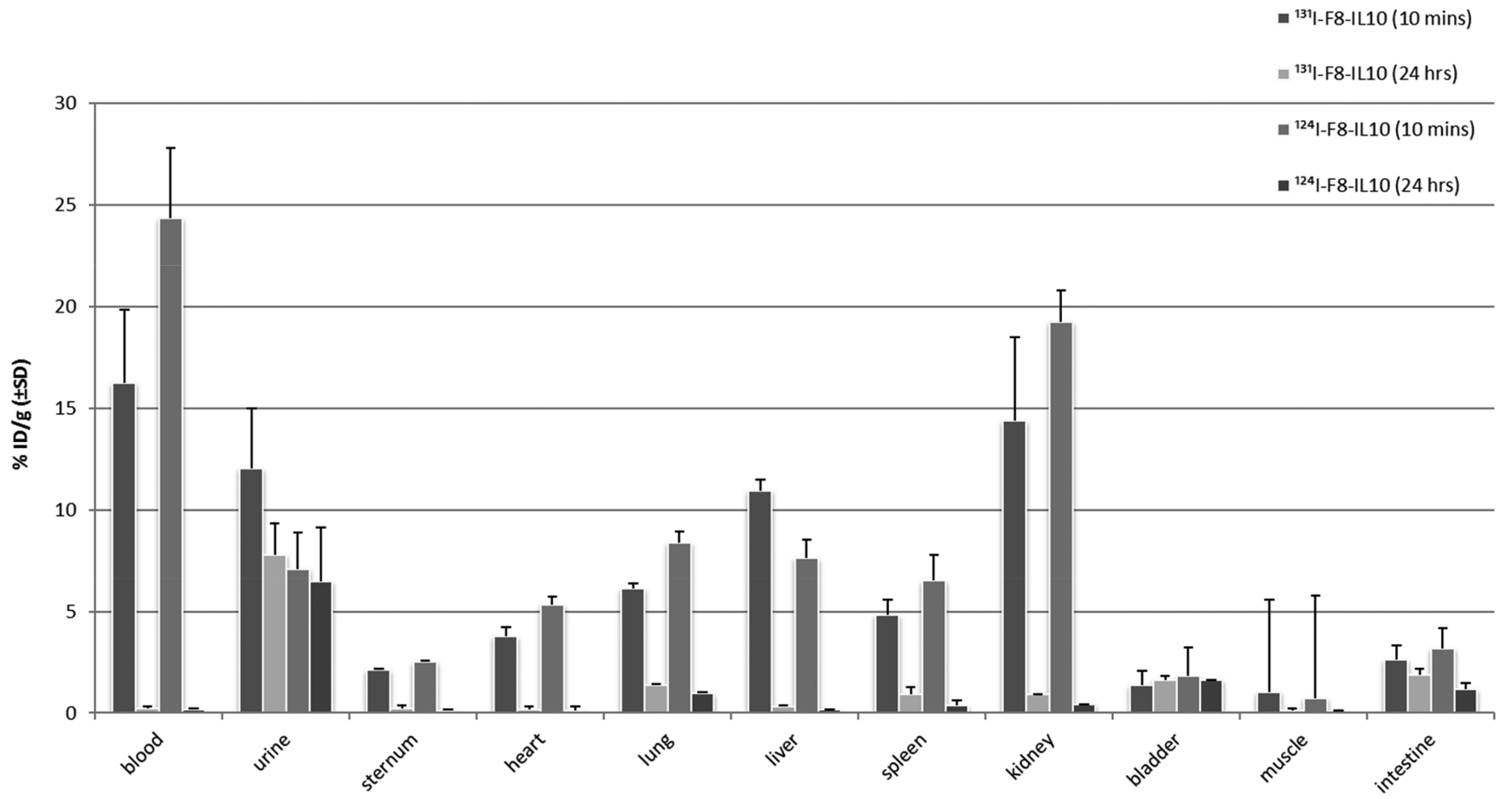

Figure 3. Ex vivo tissue distribution of healthy mice with $\left[{ }^{131} \mathrm{I}\right] \mathrm{I}-\mathrm{F} 8-\mathrm{IL} 10$ (iodogen) and $\left[{ }^{124} \mathrm{I}\right]$-F8-IL10 (chloramine-T) at $10 \mathrm{~min}$ and $24 \mathrm{~h}$. The results are expressed as a percentage of the injected dose per gram $(\% \mathrm{ID} / \mathrm{g} \pm \mathrm{SD})$.

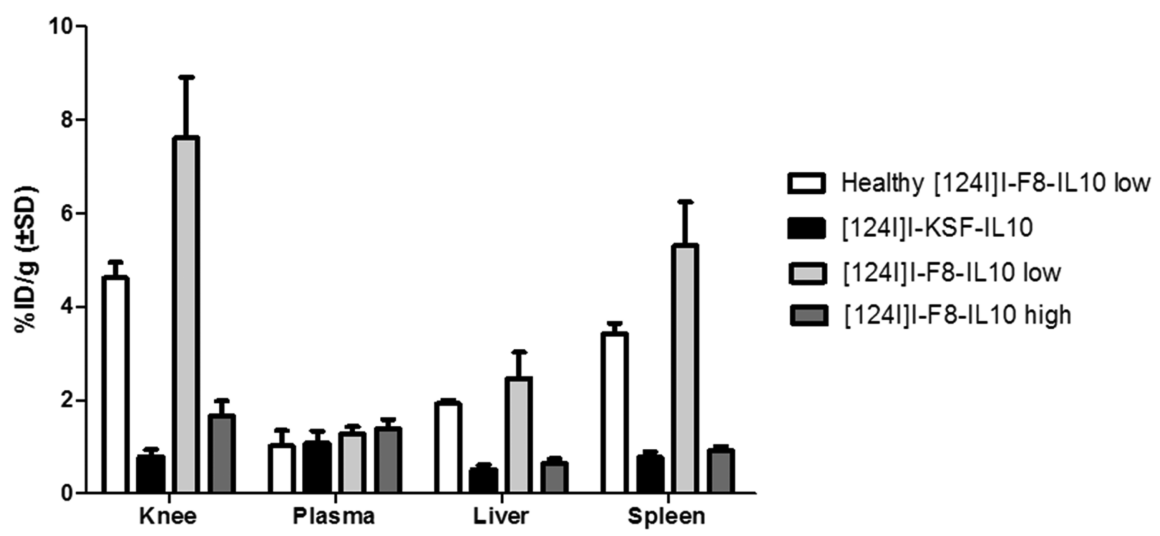

Figure 4. Ex vivo tissue distribution at $24 \mathrm{~h}$ p.i. in healthy rats, $\left[{ }^{124} \mathrm{I}\right] \mathrm{I}-\mathrm{F} 8-\mathrm{IL} 10^{\text {low }}$ (white bars), and in arthritic rats, $\left[{ }^{124} \mathrm{I}\right] \mathrm{I}-\mathrm{KSF}-\mathrm{IL} 10-\mathrm{control}$ (black bars), $\left[{ }^{124} \mathrm{I}\right] \mathrm{I}-\mathrm{F} 8-\mathrm{IL} 10^{\text {low }}$ (light gray bars), and $\left[{ }^{124} \mathrm{I}\right] \mathrm{I}-\mathrm{F} 8-\mathrm{IL} 10^{\text {high }}$ (dark gray bars). The results are expressed as percentage injected dose per gram $(\% \mathrm{ID} / \mathrm{g} \pm \mathrm{SD})$.

tracers had comparable blood levels of $16-25 \% \mathrm{ID} / \mathrm{g}$, decreasing to low values, $<0.3 \% \mathrm{ID} / \mathrm{g}$, at $24 \mathrm{~h}$ p.i. Biodistribution in various organ tissues was also similar over time, with no accumulation in liver or spleen at $24 \mathrm{~h}$. All tissues showed clearance of both tracers within the time span of $24 \mathrm{~h}$. Urine excretion was similar.

Ex Vivo Biodistribution of ${ }^{124}$ I]I-F8-IL10 in Arthritic Rats. After arthritis induction, all rats showed macroscopic thickening of the right, arthritic knee compared with the contralateral knee (data not shown). None of the rats showed any adverse effects, and no major changes in body weight were observed. Similar to observations in RA patients, uptake in arthritic knee joints was found in the F8-IL $10^{\text {low }}$ group. In this group, uptake in the arthritic knee joint of $\left[{ }^{124} \mathrm{I}\right] \mathrm{I}-\mathrm{F} 8-\mathrm{IL} 10$ was $\sim 2 \times$ higher than that $(p<0.01)$ in the (right) knee joint of a healthy (nonarthritic) rat (Figure 4). Specificity of uptake was demonstrated by a $\sim 14$-fold increased uptake of F8-IL10 in the arthritic knee joints as compared with uptake of the control antibody KSF-IL10 $(p<0.001)$. Interestingly, up to two times higher uptake of F8-IL10 was also observed in liver and spleen of arthritic rats as compared with that in healthy rats, while uptake in other organs was comparable between arthritic and healthy rats (Figure 4). When the dose of F8-IL10 was increased significantly (F8-IL10 ${ }^{\text {high }}$ group), $\left[{ }^{124} \mathrm{I}\right] \mathrm{I}-\mathrm{F} 8-\mathrm{IL} 10$ uptake decreased in several tissues with a pronounced decrease in arthritic knee joints, liver, and spleen $(p<0.01)$, while blood levels were comparable at $24 \mathrm{~h}$ p.i.

Investigation of the Effect of the Disease RA on Liver and Spleen Expression of Fibronectin ED-A. Increased expression of fibronectin ED-A in liver and spleen was observed in arthritic rats compared with healthy rats (Figure 5). In Figure 5, the white pulp (histology, Figure 5A) with fibronectin ED-A expression in the spleen of healthy (Figure 5B) and arthritic (Figure 5C) rats is shown. Fibronectin ED-A expression in spleen was $\sim 8 \times(p<0.001)$ increased in arthritic rats compared 

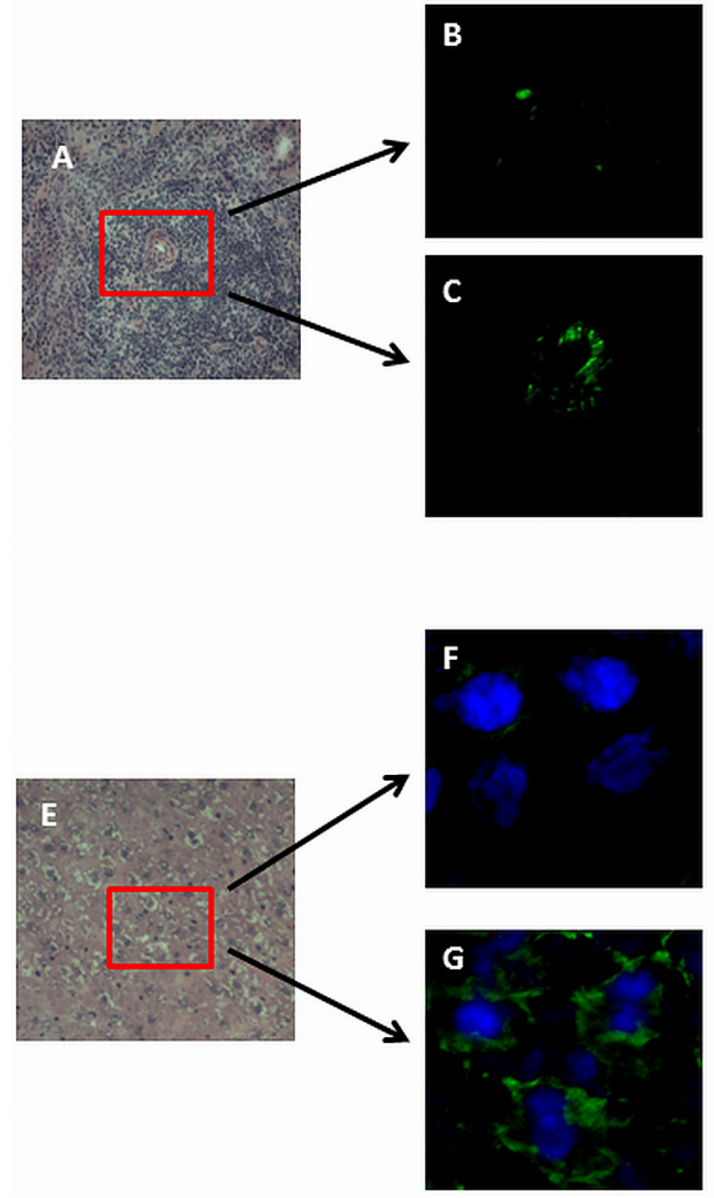

D

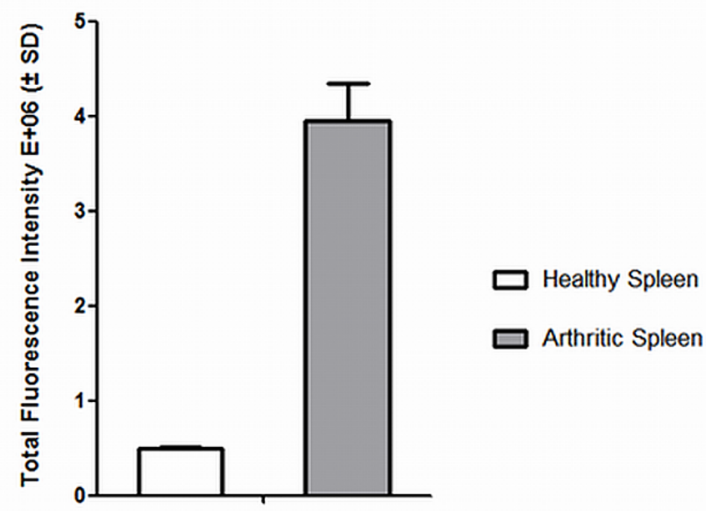

H

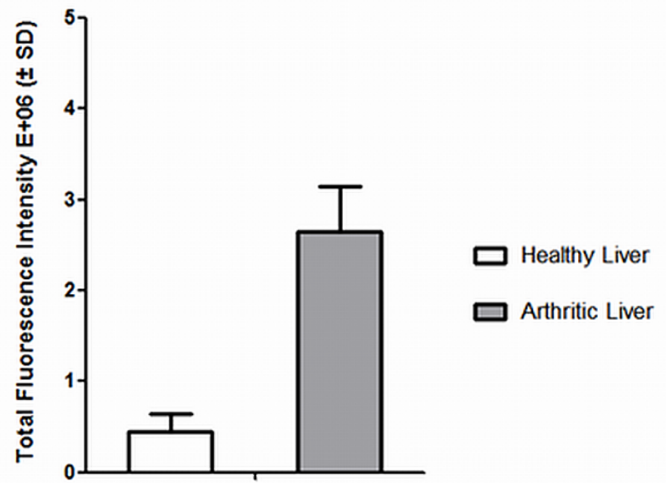

Figure 5. Histopathology and immunofluorescence images of fibronectin ED-A stainings on arthritic and healthy rats spleen and liver sections. (A) HE staining of spleen (10× magnification). (B, C) Fibronectin ED-A staining (40× magnification) of healthy spleen (B) and arthritic spleen (C). (D) The total fluorescence intensity of healthy spleen vs the arthritic spleen (total fluorescence intensity $E+06 \pm S D$ ). (E) HE staining of liver (10X magnification). (F, G) Fibronectin ED-A staining (blue channel, nucleus, and green channel, fibronectin ED-A) of healthy (F) and arthritic liver (G). $(\mathrm{H})$ The total fluorescence intensity of healthy liver vs the arthritic liver (total fluorescence intensity E+06 $\pm \mathrm{SD}$ ).

with healthy rats (Figure 5D). Further, fibronectin ED-A expression in liver (hepatocytes) (histology, Figure 5E) of healthy rats (Figure 5F) and arthritic rats (Figure 5G) was observed. Fibronectin ED-A expression in liver was $\sim 7 \times(p<$ $0.001)$ increased in arthritic rats compared with healthy rats (Figure 5H).

\section{DISCUSSION}

In this study, both targeting performance and in vivo biodistribution of the potential F8-IL10 antirheumatic drug were investigated using a translational approach. First of all, a feasibility PET-CT study in RA patients demonstrated that F8IL10 targets clinically inflamed joints and also subclinically affected joints. Similarly, targeting was also found in arthritic joints of rats. The RA patient PET study revealed unexpected in vivo biodistribution. Very rapid blood clearance (less than $1 \%$ detectable in less than $5 \mathrm{~min}$ ) and high liver and spleen uptake on PET-CT (within 1-h p.i. of $\left[{ }^{124} \mathrm{I}\right] \mathrm{I}-\mathrm{F} 8-\mathrm{IL} 10$ ) were observed. Subsequent animal experiments showed that increased tracer uptake in liver and spleen is associated with increased, arthritis related, expression of fibronectin ED-A in liver and spleen. The latter may be the main cause of local accumulation of the tracer in RA patients. In addition, a lower F8-IL10 administration dose (microdose of F8-IL10 administered in RA patients) may also result in relatively high uptake in liver and spleen, although our experiments did not allow definite conclusions for this mechanism.

Although our targeting data of F8-IL10 in arthritic joints were found in a small study of only 3 RA patients, these observations were supported by our arthritic rat data and are in line with targeting results previously found in a collagen-induced mouse model. ${ }^{16}$ This provides further evidence of the feasibility of targeted delivery of IL10 by binding to fibronectin ED-A in arthritic joints, also reflected by the promising initial phase $\mathrm{Ib}$ therapeutic efficacy data of F8-IL10 in RA patients. ${ }^{17,35}$

The observed high uptake in liver (and to some extent in spleen), as well as the fast blood clearance of $\left[{ }^{124} \mathrm{I}\right] \mathrm{I}-\mathrm{F} 8-\mathrm{IL} 10$ in RA patients, was unexpected. In previous experiments in tumorbearing mice, no increased F8-IL10 uptake in liver and spleen was observed, and blood clearance was clearly slower shortly after i.v. injection. ${ }^{15}$ There are several potential explanations for the observed differences, ( 1 ) the use of different radioiodination methods (i.e., chloramine-T method in tumor-bearing mice and iodogen in RA patients), (2) effect of the RA disease on fibronectin ED-A expression in liver and spleen, (3) different injected doses of F8-L10, and (4) species differences.

First, the potential effect of the radioiodination method on the ex vivo biodistribution was addressed in healthy mice. F8-IL10 can potentially be modified by oxidation during the radioiodination procedure, which could result in rapid blood 
clearance and uptake in the mononuclear phagocyte system (liver/spleen). ${ }^{36}$ However, no differences in liver and spleen uptake of F8-IL10 were found between both radioiodination methods. In fact, in healthy mice, liver and spleen uptake at $24 \mathrm{~h}$ p.i. were low, regardless of the radioiodination method. In addition, initial blood clearance was much slower (in line with previous observations in tumor-bearing mice ${ }^{16}$ ), with $15-25 \%$ $\mathrm{ID} / \mathrm{g}$ present at $10 \mathrm{~min}$ p.i. in healthy mice as compared to $<1 \%$ ID/L in the blood compartment of RA patients at 5 min p.i.

Second, hepatic and splenic F8-IL10 uptake of arthritic rats was more than twice as high as that in healthy rats. A high dose of F8-IL10 significantly decreased liver and spleen uptake of $\left[{ }^{124} \mathrm{I}\right] \mathrm{I}-\mathrm{F} 8-\mathrm{IL} 10$ in rats with experimental arthritis, indicating specific binding in these tissues. To explore whether this observation could be explained by increased fibronectin ED-A expression and hence increased F8-IL10 binding in arthritic rats, further immunohistochemical analyses were performed. Indeed, in arthritic rats, significantly higher fibronectin ED-A expression was found in liver and spleen as compared with healthy rats.

Third, a microdose of i.v.-administered agent may directly accumulate in high antigen expressing and well accessible organs upon the first passage through these organs (liver/spleen). This so-called antigen sink theory has also been observed by others in B-cell lymphoma patients injected with radiolabeled rituximab. ${ }^{37}$ The RA patients were injected with a 500-fold lower dose of F8-IL10 than healthy mice ( 0.006 versus $3 \mathrm{mg} / \mathrm{kg}$ F8IL10), where no accumulation of radioactivity in liver and spleen was observed. Thus, in the patient study, liver and spleen may have acted as antigen sinks for the low dosage of i.v.administered F8-IL10, which could explain the fast blood clearance and relatively low uptake in arthritic joints. Upon increase of the F8-IL10 dose, liver and spleen may become saturated, resulting in higher plasma concentration, thereby leaving relatively more F8-IL10 available to bind to inflamed joints. To investigate this antigen sink theory, a high dose of F8IL10 ( 2000-fold higher than that in humans) in arthritic rats was compared with the lowest feasible F8-IL10 dose for administration in rats ( $\sim 50$-fold fold higher than in humans). Indeed, an uptake of radiolabeled F8-IL10 in the liver/spleen of the F8-IL10 high group was significantly lower than that seen in the F8-IL10 ${ }^{\text {low }}$ group. However, uptake of radioactive F8-IL10 in several other tissues was decreased as well (data not shown), which presumably was, at least in part, related to blockade of local F8-IL10 binding sites. Therefore, these results could not definitely prove or rule out a possible role of liver and spleen as antigen sinks in the case of the F8-IL $10^{\text {low }}$ dose.

Finally, species differences between animals and humans cannot be ruled out. Findings in animals can be highly suggestive, but extrapolation to humans should be made with care. In the present study, direct dose comparisons were not possible since lowering the F8-IL10 dose $(<0.3 \mathrm{mg} / \mathrm{kg})$ in rats to allow for a direct comparison with the dose in RA patients $(0.006 \mathrm{mg} / \mathrm{kg})$ was technically not possible. Apart from tissue observations, differences in pharmacokinetics, stability of the tracer, and targeting between animals and patients may also play a role.

\section{CONCLUSIONS}

In conclusion, the imaging studies demonstrate that PET-guided drug development may provide significant information on the targeting potential of a new antirheumatic drug, although observations were made in a small group of 3 RA patients. $\left[{ }^{124} \mathrm{I}\right] \mathrm{I}-\mathrm{F} 8-\mathrm{IL} 10 \mathrm{PET}$ revealed promising targeting in arthritic joints in RA patients. In addition, PET whole body biodistribution data may point at tracer accumulation at other nontarget sites for F8-IL10, likely related to disease-induced antigen expression at these sites and possibly related to antibody dosing. These insights can add to optimal dosing schedules for targeting sites of interest as well as inform on potential sites of concern for side effects. So far, the human phase $1 \mathrm{~b}$ data have not revealed major side effects. F8-IL10 was generally well tolerated up to a dose of $600 \mu \mathrm{g} / \mathrm{kg}^{35}$

\section{ASSOCIATED CONTENT}

\section{S Supporting Information}

The Supporting Information is available free of charge on the ACS Publications website at DOI: 10.1021/acs.molpharmaceut.8b00982.

Complete details of tracer synthesis for patients, mice, and rat studies (PDF)

Table with baseline patient demographics and clinical and functional characteristics of three RA patients (PDF)

\section{AUTHOR INFORMATION}

\section{Corresponding Author}

*Phone: +31 20444 4434; Fax: +31 20444 2138; E-mail: j. vanderlaken@vumc.nl.

\section{ORCID}

Stefan T.G. Bruijnen: 0000-0003-2155-4929

\section{Present Address}

\#GSK Vaccines SrL, via Fiorentina 1, 53100 Siena, Italy

\section{Author Contributions}

S.T.G.B. and D.M.S.H.C. contributed equally to this work. S.T.G.B. and D.S.M.H.C. contributed to the acquisition, analysis, and interpretation of the data of human and animal data, respectively, and drafted the manuscript. C.F.M.M., G.J., and C.J.vdL. were involved with design and interpretation of all studies and critically revised the manuscript. C.F.M.M., G.J., O.S.H., C.J.vdL., and G.A.M.S.vD. contributed to the design of the study and the interpretation of the human data and critically revised the manuscript. D.J.V. was involved in radiolabeling of the compounds. A.A.L. contributed to the interpretation of the PET-CT data and critically revised the manuscript. R.J.P.M. contributed to analysis and the interpretation of the histological immunofluorescence data. L.G. and D.N. contributed to the design of the experiments and critical review of the data.

\section{Notes}

Ethics approval and consent to participate. The study protocol was approved by the VU University Medical Center Medical Ethics Review Committee. All patients gave written informed consent prior to participation in the study. All animal experiments were performed in accordance with the European Community Council Directive 2010/63/EU for laboratory animal care and the Dutch Law on animal experimentation. The experimental protocol was approved by the local committee on animal experimentation of the VU University Medical Center. The authors declare no competing financial interest.

\section{ACKNOWLEDGMENTS}

This work was funded by the FP7-Health Innovation and was part of the grant "Profiling Responders In Antibody Therapies" (PRIAT). We gratefully acknowledge $M$. van Dijk- Baak (research nurse) for excellent patient care during the study; Thijs Nell and Ricardo Vos for labeling; Ricardo Vos, Mariska 
Verlaan, and Elise Mantel for excellent technical support and animal experiments; Jeroen Kole for helping with data acquisition and analysis of the immunofluorescence data; and Maqsood Yaqub for help with quantification of PET scans. The data sets used and/or analyzed during the current study are available from the corresponding author upon reasonable request.

\section{ABBREVIATIONS}

RA, rheumatoid arthritis; DMARD, disease-modifying antiinflammatory drugs; F8, F8 antibody fibronectin; IL10, interleukin 10; PET-CT, positron emission tomographycomputer tomography; SD, standard deviation; ID/g, injected dose/gram; \%ID, percentage of injected dose; \%ID/L, percentage injected dose per liter; ED-A, extra-domain-A of fibronectin; p.i., post injection; iTLC, instant thin layer chromatography; HPLC, high-performance liquid chromatography.

\section{REFERENCES}

(1) McInnes, I. B.; Schett, G. The pathogenesis of rheumatoid arthritis. N. Engl. J. Med. 2011, 365 (23), 2205-19.

(2) Smolen, J. S.; Landewe, R.; Breedveld, F. C.; Dougados, M.; Emery, P.; Gaujoux-Viala, C.; et al. EULAR recommendations for the management of rheumatoid arthritis with synthetic and biological disease-modifying antirheumatic drugs. Ann. Rheum. Dis. 2010, 69, 964-75.

(3) van Schouwenburg, P. A.; Rispens, T.; Wolbink, G. J. Immunogenicity of anti-TNF biologic therapies for rheumatoid arthritis. Nat. Rev. Rheumatol. 2013, 9 (3), 164-72.

(4) Smolen, J. S.; Aletaha, D.; McInnes, I. B. Rheumatoid arthritis. Lancet 2016, 388, 2023-38.

(5) McInnes, I. B.; Liew, F. Y. Cytokine networks-towards new therapies for rheumatoid arthritis. Nat. Clin. Pract. Rheumatol. 2005, 1 (1), 31-9.

(6) Sauer, S.; Erba, P.; Petrini, M.; et al. Expression of the oncofetal ED-B - containing fibronectin isoform in hematologic tumors enables ED-B - targeted 131 I-L19SIP radioimmunotherapy in Hodgkin lymphoma patients. Blood. 2009, 113, 2265-2274.

(7) King, D. M.; Albertini, M. R.; Schalch, H.; Hank, J. A.; Gan, J.; Surfus, J.; et al. Phase I clinical trial of the immunocytokine EMD 273063 in melanoma patients. J. Clin. Oncol. 2004, 22, 4463-73.

(8) Mårlind, J.; Kaspar, M.; Trachsel, E.; Sommavilla, R.; Hindle, S.; Bacci, C.; et al. Antibody-mediated delivery of interleukin-2 to the stroma of breast cancer strongly enhances the potency of chemotherapy. Clin. Cancer Res. 2008, 14, 6515-24.

(9) Pedretti, M.; Verpelli, C.; Mårlind, J.; Bertani, G.; Sala, C.; Neri, D.; et al. Combination of Temozolomide with immunocytokine F16IL2 for the treatment of glioblastoma. Br. J. Cancer 2010, 103, 827-36.

(10) Ip, W. K. E.; Hoshi, N.; Shouval, D. S.; Snapper, S.; Medzhitov, R. Anti-inflammatory effect of IL-10 mediated by metabolic reprogramming of macrophages. Science 2017, 356, 513 LP-9. .

(11) Murray, P. J. The primary mechanism of the IL-10-regulated antiinflammatory response is to selectively inhibit transcription. Proc. Natl. Acad. Sci. U. S. A. 2005, 102, 8686-91.

(12) Weinblatt, M. E., St; Clair, E. W.; Breedveld, F. C.; Moreland, L. W.; Keystone, E. C.; Lee, S. H.; et al. RHUIL-10 (TENOVIL) plus methotrexate (MTX) in active rheumatoid arthritis (RA): A phase I/II study. Arthritis Rheum. 1999, 42 (9), S170.

(13) van Beers, J. J.; Willemze, A.; Stammen-Vogelzangs, J.; Drijhout, J. W.; Toes, R. E.; M Pruijn, G. J. Anti-citrullinated fibronectin antibodies in rheumatoid arthritis are associated with human leukocyte antigen-DRB1 shared epitope alleles. Arthritis Research \& Therapy 2012, 14, R35.

(14) Rybak, J. N.; Roesli, C.; Kaspar, M.; Villa, A.; Neri, D. The extradomain A of fibronectin is a vascular marker of solid tumors and metastases. Cancer Res. 2007, 67, 10948-57.
(15) Villa, A.; Trachsel, E.; Kaspar, M.; Schliemann, C.; Sommavilla, R.; Rybak, J. N.; et al. A high-affinity human monoclonal antibody specific to the alternatively spliced EDA domain of fibronectin efficiently targets tumor neo-vasculature in vivo. Int. J. Cancer 2008, 122, 2405-13.

(16) Schwager, K.; Kaspar, M.; Bootz, F.; Marcolongo, R.; Paresce, E.; Neri, D.; et al. Preclinical characterization of DEKAVIL (F8-IL10), a novel clinical-stage immunocytokine which inhibits the progression of collagen-induced arthritis. Arthritis Research \& Therapy 2009, 11, R142.

(17) Galeazzi, M.; Bazzichi, L.; Sebastiani, G. D.; Neri, D.; Garcia Gonzalez, E.; Ravenni, N.; et al. A phase IB clinical trial with dekavil (F8-Il10), An immunoregulatory armed antibody for the treatment of rheumatoid arthritis, used in combination with methotrexate. Israel Medical Association Journal 2014, 16, 666.

(18) Mammatas, L. H.; Verheul, H. M. W.; Hendrikse, N. H.; Yaqub, M.; Lammertsma, A. A.; Menke-van der Houven van Oordt, C. W. Molecular imaging of targeted therapies with positron emission tomography: the visualization of personalized cancer care. Cell. Oncol. 2015, 38, 49-64.

(19) Mankoff, D. A.; Farwell, M. D.; Clark, A. S.; Pryma, D. A. Making Molecular Imaging a Clinical Tool for Precision Oncology: A Review. JAMA Oncol. 2017, 3 (5), 695-701.

(20) Robinson, M. K.; Doss, M.; Shaller, C.; Narayanan, D.; Marks, J. D.; Adler, L. P.; et al. Quantitative immuno-positron emission tomography imaging of HER2-positive tumor xenografts with an iodine-124 labeled anti-HER2 diabody. Cancer Res. 2005, 65 (4), 1471-8.

(21) Van Dongen, G. A. M. S.; Huisman, M. C.; Boellaard, R.; Hendrikse, N. H.; Windhorst, A. D.; Visser, G. W. M.; et al. 89Zrimmuno-PET for imaging of long circulating drugs and disease targets: Why, how and when to be applied? Quarterly Journal of Nuclear Medicine and Molecular Imaging 2015, 59, 18-38.

(22) van Dongen, G. A. M. S.; Visser, G. W. M.; Lub-de Hooge, M. N.; de Vries, E. G.; Perk, L. R. Immuno-PET: A Navigator in Monoclonal Antibody Development and Applications. Oncologist 2007, 12, 13791389.

(23) Arnett, F. C.; Edworthy, S. M.; Bloch, D. A.; McShane, D. J.; Fries, J. F.; Cooper, N. S.; et al. The American Rheumatism Association 1987 revised criteria for the classification of rheumatoid arthritis. Arthritis Rheum. 1988, 31, 315-24.

(24) Chandrupatla, D. M. S. H.; Weijers, K.; Gent, Y.Y.J.; De Greeuw, I.; Lammertsma, A. A.; Jansen, G.; et al. Sustained macrophage infiltration upon multiple intra-articular injections: An improved rat model of rheumatoid arthritis for PET guided therapy evaluation. BioMed Res. Int. 2015, 2015, 2015.

(25) Chandrupatla, D.; Jansen, G.; Mantel, E.; Low, P. S.; Matsuyama, T.; Musters, R. P.; et al. Imaging and Methotrexate Response Monitoring of Systemic Inflammation in Arthritic Rats Employing the Macrophage PET Tracer [(18)F]Fluoro-PEG-Folate. Contrast Media Mol. Imaging 2018, 2018, 1.

(26) Lindmo, T.; Boven, E.; Cuttitta, F.; Fedorko, J.; Bunn, P. A., Jr. Determination of the immunoreactive fraction of radiolabeled monoclonal antibodies by linear extrapolation to binding at infinite antigen excess. J. Immunol. Methods 1984, 72 (1), 77-89.

(27) Erba, P. A.; Sollini, M.; Orciuolo, E.; Traino, C.; Petrini, M.; Paganelli, G.; et al. Radioimmunotherapy with Radretumab in Patients with Relapsed Hematologic Malignancies. J. Nucl. Med. 2012, 53, 9227.

(28) Tijink, B. M.; Perk, L. R.; Budde, M.; Stigter-Van Walsum, M.; Visser, G. W. M.; Kloet, R. W.; et al. 124I-L19-SIP for immuno-PET imaging of tumour vasculature and guidance of131I-L19-SIP radioimmunotherapy. Eur. J. Nucl. Med. Mol. Imaging 2009, 36, 1235-44.

(29) Frey, K.; Zivanovic, A.; Schwager, K.; Neri, D. Antibody-based targeting of interferon-alpha to the tumor neovasculature: a critical evaluation. Integr Biol. (Camb). 2011, 3 (4), 468-78.

(30) Boellaard, R.; Delgado-Bolton, R.; Oyen, W. J.; Giammarile, F.; Tatsch, K.; Eschner, W.; et al. FDG PET/CT: EANM procedure guidelines for tumour imaging: version 2.0. Eur. J. Nucl. Med. Mol. Imaging 2015, 42 (2), 328-54. 
(31) Boellaard, R.; Oyen, W. J. G.; Hoekstra, C. J.; Hoekstra, O. S.; Visser, E. P.; Willemsen, A. T.; et al. The Netherlands protocol for standardisation and quantification of FDG whole body PET studies in multi-centre trials. Eur. J. Nucl. Med. Mol. Imaging 2008, 35, 2320-33. (32) Krak, N. C.; Boellaard, R.; Hoekstra, O. S.; Twisk, J. W. R.; Hoekstra, C. J.; Lammertsma, A. A. Effects of ROI definition and reconstruction method on quantitative outcome and applicability in a response monitoring trial. Eur. J. Nucl. Med. Mol. Imaging 2005, 32, 294-301.

(33) Vanderhoek, M.; Perlman, S. B.; Jeraj, R. Impact of the definition of peak standardized uptake value on quantification of treatment response. J. Nucl. Med. 2012, 53 (1), 4-11.

(34) Verbrugge, S. E.; Al, M.; Assaraf, Y. G.; Kammerer, S.; Chandrupatla, D. M. S. H.; Honeywell, R.; et al. Multifactorial resistance to aminopeptidase inhibitor prodrug CHR2863 in myeloid leukemia cells: down-regulation of carboxylesterase 1, drug sequestration in lipid droplets and pro-survival activation ERK/Akt/mTOR. Oncotarget 2016, 7, 5240-57.

(35) Galeazzi, M.; Sebastiani, G.; Voll, R.; Wollenhaupt, J.; Viapiana, O.; Dudler, J.; et al. OP0099 Safety, tolerability and initial signs of efficacy of the fully human immunocytokine DEKAVIL (F8IL10): a novel therapeutic approach for rheumatoid arthritis. Ann. Rheum Dis. 2017, 76, 92 LP-3. .

(36) Sobal, G.; Resch, U.; Sinzinger, H. Modification of low-density lipoprotein by different radioiodination methods. Nucl. Med. Biol. 2004, 31 (3), 381-8.

(37) Muylle, K.; Flamen, P.; Vugts, D. J.; Guiot, T.; Ghanem, G.; Meuleman, N.; et al. Tumour targeting and radiation dose of radioimmunotherapy with 90Y-rituximab in CD20+ B-cell lymphoma as predicted by $89 \mathrm{Zr}$-rituximab immuno-PET: impact of preloading with unlabelled rituximab. Eur. J. Nucl. Med. Mol. Imaging 2015, 42, 1304-14. 